\title{
Effect of Duration of Silicone Oil Tamponade on Retinal Structure after Rhegmatogenous Retinal Detachment Surgery
}

\author{
Chloé Dubroux ${ }^{a} \quad$ Julia Salleron ${ }^{b} \quad$ Karine Angioi-Duprez ${ }^{a} \quad$ Jean-Paul Berrod ${ }^{a}$ \\ Jean-Baptiste Conart ${ }^{a}$ \\ ${ }^{a}$ Department of Ophthalmology, Nancy University Hospital, Vandoeuvre-lès-Nancy, France; b Institut de \\ Cancérologie de Lorraine, Department of Biostatistics and Data Management, Université de Lorraine, \\ Vandoeuvre-lès-Nancy, France
}

\section{Keywords}

Macular thickness - Optical coherence tomography · Rhegmatogenous retinal detachment · Silicone oil · Vitreoretinal surgery

\begin{abstract}
Purpose: The aim of the study was to investigate the effect of tamponade duration on retinal changes induced by silicone oil (SO) in patients who underwent successful rhegmatogenous retinal detachment (RRD) surgery. Methods: Retrospective comparative case series of 68 patients who underwent SO tamponade for RRD. Patients were divided into 2 groups based on timing of SO removal: $<6$ months (group $1, n=34$ ) versus $\geq 6$ months (group $2, n=34$ ). The main outcome measure was the change in central macular, inner, and outer retinal layer thickness (CMT, IRLT, and ORLT) before and after SO removal (SOR). Results: The median tamponade duration was 4 [Clin Ophthalmol. 2016;10:471-6, Zhonghua Yan Ke Za Zhi. 1997 Jan;33(1):39-41] months in group 1 and 8 [Arch Ophthalmol. 1994 Jun;112(6):778-85, Retina. $2004 \mathrm{Dec} ; 24(6): 871-7]$ months in group 2 ( $p<0.001)$. The mean CMT significantly increased from $245.3 \pm 22.2 \mu \mathrm{m}$ and $238.8 \pm 41.6 \mu \mathrm{m}$ under SO to $281.3 \pm 60.2 \mu \mathrm{m}$ and 259.0
\end{abstract}

karger@karger.com www.karger.com/oph

Karger $\frac{1}{\%}$
(C) 2021 The Author(s)

Published by S. Karger AG, Basel

This is an Open Access article licensed under the Creative Commons Attribution-NonCommercial-4.0 International License (CC BY-NC) (http://www.karger.com/Services/OpenAccessLicense), applicable to the online version of the article only. Usage and distribution for commercial purposes requires written permission. $\pm 43.5 \mu \mathrm{m}$ after SOR in group $1(p=0.009)$ and in group 2 $(p=0.007)$, respectively. Automated segmentation measurement revealed a significant increase in mean IRLT ( $p=0.014$ and $p=0.013$ ) but no change in mean ORLT ( $p=0.080$ and $p=0.257$ ) in both groups. After adjustment, there was no difference between the 2 groups in terms of mean final CMT, IRLT, and ORLT and mean retinal thickness changes after SOR. There was also no correlation between the tamponade duration and macular microstructural changes or visual recovery. Conclusion: SO tamponade causes a thinning of all retinal layers, mainly affecting the inner retinal layer. However, these changes resolved following SO extraction and were not affected by longer tamponade duration.

(C) 2021 The Author(s).

Published by S. Karger AG, Basel

\section{Introduction}

Silicone oil (SO) is widely used as an intraocular tamponade agent for the management of complex retinal diseases such as advanced proliferative diabetic retinopathy, ocular trauma, and rhegmatogenous retinal detachment (RRD) associated with severe proliferative vitreoretinop- 
athy (PVR) or giant retinal tear [1,2]. As a chemically inert substance with high surface tension and viscosity, it provides excellent structural support to keep the retina attached for an extended period of time [3]. However, its use is generally intended to be temporary as prolonged tamponade may lead to anterior segment complications, including oil emulsification, cataract, increased intraocular pressure (IOP), secondary glaucoma, and band keratopathy $[3,4]$. The optimal timing for SO removal (SOR) is unknown, but many surgeons agree that $\mathrm{SO}$ should be removed within 3-6 months after its injection to minimize these complications $[5,6]$.

Mild visual loss may also occur following SOR due to postoperative hypotony, cystoid macular oedema (CME), or development of epiretinal membranes (ERMs) [7, 8]. Nevertheless, some patients may experience sudden deterioration in central vision without any apparent explanation $[9,10]$. This phenomenon can occur either during SO tamponade or after SOR with a reported incidence of $1-29.7 \%[8,9]$. The pathophysiology of this dramatic condition remains elusive although electrophysiologic studies have provided evidence of both macular and optic nerve dysfunction [11]. Interestingly, Christensen and la Cour revealed that unexplained severe visual loss in SOoperated eyes was associated with a significant thinning of inner retinal layers, suggesting neuronal cell loss in the macular area as a possible explanation [12].

Numerous studies have subsequently looked into the effect of SO tamponade on macular anatomy and reported contradictive results regarding the change in retinal layer thickness and the layers affected [13-19]. The reasons for these discrepancies are unclear but may be related to differences in study design, inclusion criteria, methods of evaluation, and time of assessment after surgery.

In contrast, only a few studies specifically investigated the relationship between the tamponade duration and SO-associated retinal changes $[2,14-16,18,20-23]$. Some authors reported that eyes with longer term tamponade were more likely to exhibit outer retinal layer (ORL) damage and worse visual acuity after SOR while others did not find any difference in the degree of retinal thinning or vision loss [16, 20-23]. Thus, it is still not clear whether or not early SOR may result in improved anatomical and functional outcomes.

The aim of this study was to examine the effect of tamponade duration on $\mathrm{SO}$-associated retinal changes in patients who underwent successful RRD surgery. We also assessed the correlation between the tamponade period and final visual acuity.

Effect of Silicone Oil Tamponade on

Retina after RRD Surgery

\section{Methods}

Patients and Study Design

A retrospective review of medical records was performed on 832 consecutive patients who underwent pars plana vitrectomy (PPV) for RD at the University Hospital of Nancy from April 2015 to October 2019. All of them were given complete information on the risks and benefits of the surgical procedure and gave their written consent before surgery. The study adhered to the tenets of the Declaration of Helsinki, and the protocol was approved by the Ethics committee of the French Society of Ophthalmology.

Inclusion criteria were as follows: (1) patients with successfully repaired RRD by PPV and SO tamponade; (2) a minimum followup period of 1 month after SOR. Patients were excluded from the analysis if they had: (1) non-rhegmatogenous $\mathrm{RD},(2)$ traumatic $\mathrm{RRD}$, (3) RRD with severe anterior PVR requiring peripheral retinectomy, (4) retinal redetachment during SO tamponade, (5) prior history of SO tamponade, (6) pre-existing macular pathologic features, such as macular hole, age-related macular degeneration, diabetic maculopathy, (7) unavailable or low-quality and unreliable postoperative OCT images.

All patients underwent a detailed ophthalmologic examination preoperatively and at each follow-up visit including best-corrected visual acuity (BCVA) measured with projected-light Snellen charts, IOP measurement using a non-contact tonometer, axial length (AL) measurement using IOL Master (IOL Master; Carl Zeiss Meditec AG, Jena, Germany), biomicroscopy with anterior segment evaluation and fundus. An Amsler-Dubois scheme was systematically established for each patient, specifying the extent of the RD, number and location of retinal breaks, existence of vitreous haemorrhage, and preoperative PVR grading according to Machemer et al. [24].

Patients were divided into 2 groups based on the timing of SOR: group 1 consisting of patients who underwent SO extraction within 6 months of RRD surgery and group 2 consisting of patients who underwent $\mathrm{SO}$ extraction $>6$ months after RRD surgery. The timing for SOR was at the surgeons' discretion according to prior history, condition of the contralateral eye and patient preferences.

Patients were examined within the first week after RRD surgery and at various times thereafter. A full ophthalmologic examination including macular imaging using the Spectralis HRA-OCT (Heidelberg Engineering, Heidelberg, Germany) was systematically carried out in the month prior to SOR and at least 1 month after SO extraction.

\section{Surgical Procedure}

All procedures were performed by 2 experienced surgeons (J.P.B. and J.B.C.). All patients underwent an extensive 3-port PPV using 23-gauge instrumentation (EVA phacovitrectomy system, DORC, Dutch Ophthalmic Research Corporation, Zuidland, The Netherlands) followed by removal of any ERMs. The internal limiting membrane (ILM) was systematically peeled off the posterior pole to the major arcade vessels after Brilliant Blue $G$ staining (ILM-Blue $^{\circledR}$, DORC, Zuidland, The Netherlands) in eyes with extensive PVR involving the macula. The decision to use perfluorocarbon liquid (PFCL) was left to the surgeon's discretion. Fluid-air exchange was then performed, followed by retinopexy with either endophotocoagulation or cryotherapy and SO was injected within the vitreous cavity. In cases with giant retinal tear, PFCL was directly exchanged with SO. SO with viscosity of $1,000 \mathrm{cST}$ (Purified 
SO 1,000 cSt in syringe, FCI, Besançon, Franche-Comté, France) was used in all patients. Combined phacoemulsification with posterior chamber intraocular implantation was performed prior to the PPV in eyes with visually significant cataract.

$\mathrm{SO}$ was extracted through 23 -gauge pars plana sclerotomy with active drainage followed by fluid-air exchange to remove residual SO droplets. Combined cataract extraction and ERM peeling were carried out as needed.

All patients received topical anti-inflammatory and antibiotic treatments for 4 weeks after RRD surgery and SOR. IOP-lowering medication (carbonic anhydrase inhibitor/beta-blocker combination) was systematically administered during the period of SO tamponade.

\section{Macular Imaging with SD-OCT}

Microstructural imaging analysis of the fovea was performed using Spectralis HRA-OCT (Heidelberg Engineering Spectralis, Heidelberg, Germany). The protocol consisted of 2 high-resolution horizontal and vertical scans, ART5, of $6 \mathrm{~mm}$ and a volume scan using 25 equally spaced horizontal B-scans centred on the fovea and covering an area of $20^{\circ}$ horizontally and vertically.

Central macular thickness (CMT) was measured using the built-in Spectralis software. Automated segmentation was then applied to distinguish inner retinal layers (distance between the ILM and the external limiting membrane [ELM]) from ORLs (distance between the ELM and the retinal pigmentary epithelium) and quantify their thickness. In case of misalignment, the segmentation was adjusted manually. The ELM and the ellipsoid zone (EZ) were defined as the third and second hyperreflective bands above the retinal pigment epithelium, according to the classification proposed by the International Nomenclature for Optical Coherence Tomography panel [25].

Their integrity was assessed as follows: line not visible or disrupted in at least 1 scan (band defect) and continuous line in both the horizontal and vertical scans (intact band). The same analysis was performed in the fellow eyes at each follow-up visit to minimize potential bias related to artefacts.

Postoperatively, the ELM or EZ were considered to be recovered if the band was either invisible or disrupted at 1 visit and intact at the next visit on the same follow-up scans. All qualitative OCT evaluations and measurements were performed independently by 2 masked readers (C.D. and J.B.C.), both unaware of the patients' visual outcomes.

Pre-, Intra-, and Postoperative Data

Pre- and intraoperative data included patient age and sex, AL, lens status, BCVA, characteristics of RRD and surgical manoeuvres during PPV and SOR (combined cataract extraction, use of PFCL, ILM, or ERM peeling).

Standard deviation (SD)-OCT findings, including central macular, inner, and ORL thicknesses (CMT, IRLT, and ORLT), integrity of the ELM and the EZ, presence of persistent subfoveolar fluid (PSF), development of CME and ERM and complications, were gathered during the period of tamponade. The following data were collected after SOR: median follow-up duration, rate of retinal redetachment, final BCVA, SD-OCT findings, and complications.

\section{Main Outcome Measures}

The main outcome measure was the change in CMT, IRLT, and ORLT before and after SO extraction. Eyes with ERM, CME, and
PSF were not considered for this analysis. Secondary outcomes were macular microstructural changes, complication rates, and final visual acuity.

\section{Statistical Analysis}

The qualitative parameters were described as frequency and percentage, and the quantitative parameters were described as mean and SD or median and interquartile range. The normality of the distribution was investigated with the Shapiro-Wilk test. Comparisons between the 2 groups were performed using the $\chi^{2}$ test or Fisher's exact test for qualitative parameters and the Student's $t$ test or Mann-Whitney U test for quantitative parameters. Comparison of CMT changes after SOR was performed using a multivariate linear regression analysis to adjust for pre-SOR values and the presence of at least 1 macular complication during the follow-up. The same analysis was applied for IRLT, ORLT, and BCVA changes. Association between tamponade duration and retinal thickness and BCVA changes was assessed using covariance analysis with tamponade duration and pre-SOR values as dependent parameters. All statistical analyses were performed using SAS software version 9.4 (SAS Institute Inc., Cary, NC, USA 25513). $p$ values $<0.05$ were considered statistically significant.

\section{Results}

During the study period, 832 patients underwent PPV for RD. Of these, 764 were excluded for the following reasons: RRD successfully repaired with gas tamponade $(n=$ $590)$, non-rhegmatogenous or traumatic $\mathrm{RD}(n=25)$, severe PVR cases requiring peripheral retinectomy $(n=18)$, retinal redetachment during SO tamponade $(n=23)$, prior history of SO tamponade $(n=44)$, pre-existing macular pathologic features $(n=31)$, absence of or low-quality postoperative SD-OCT images $(n=33)$ and follow-up period of $<1$ month after SO removal. As a result, 68 eyes of 68 patients met the inclusion criteria for the study: 34 who underwent SOR within 6 months of (group 1) and 34 who underwent SOR $>6$ months (group 2) after RRD surgery.

\section{Baseline Characteristics and Intraoperative Data}

Baseline characteristics and intraoperative data for both groups are given in Table 1. Twenty-seven (79\%) men were included in group 1 and $26(77 \%)$ in group 2 $(p=0.770)$. The mean age was $58.7 \pm 11.6$ years in group 1 and $61.0 \pm 14.2$ years in group $2(p=0.463)$. The mean $\mathrm{AL}$ in group $1(25.0 \pm 1.9 \mathrm{~mm})$ did not differ from that in group $2(24.9 \pm 1.9)(p=0.791)$.

Twelve (35\%) eyes in group 1 and $9(27 \%)$ eyes in group 2 had prior history of RRD surgery; this difference was not statistically significant $(p=0.431)$. There was also no significant difference between the 2 groups in terms of duration of $\operatorname{RRD}(p=0.487)$, extent of $\operatorname{RRD}(p=1)$, mac-
Dubroux/Salleron/Angioi-Duprez/ Berrod/Conart 
Table 1. Baseline characteristics and intraoperative data of patients who underwent SOR within 6 months of (= group 1$)$ or $>6$ months after (= group 2$)$ RRD surgery

\begin{tabular}{|c|c|c|c|}
\hline & Group $1(n=34)$ & Group $2(n=34)$ & $p$ value \\
\hline Male, $n(\%)$ & $27(79)$ & $26(77)$ & $0.770^{\mathrm{a}}$ \\
\hline Age, years $($ mean $\pm S D)$ & $58.7 \pm 11.6$ & $61.0 \pm 14.2$ & $0.463^{b}$ \\
\hline $\mathrm{AL}, \mathrm{mm}($ mean $\pm \mathrm{SD})$ & $25.0 \pm 1.9$ & $24.9 \pm 1.9$ & $0.791^{b}$ \\
\hline Prior history of RRD surgery, $n(\%)$ & $12(35)$ & $9(27)$ & $0.431^{\mathrm{a}}$ \\
\hline Duration of RRD, days (median [IQR]) & $6[3 ; 15]$ & $7[4 ; 12]$ & $0.487^{d}$ \\
\hline \multicolumn{4}{|l|}{ Lens status } \\
\hline Phakic, $n(\%)$ & $23(68)$ & $13(38)$ & \multirow{2}{*}{$0.015^{\mathrm{a}}$} \\
\hline Pseudophakic, $n$ (\%) & $11(32)$ & $21(32)$ & \\
\hline \multicolumn{4}{|l|}{ Extent of RRD } \\
\hline 1 quadrant, $n(\%)$ & $4(12)$ & $5(15)$ & \multirow{2}{*}{$1^{c}$} \\
\hline 2 quadrants or more, $n(\%)$ & $30(88)$ & $29(85)$ & \\
\hline Giant retinal tear, $n(\%)$ & $8(24)$ & $7(21)$ & $0.770^{\mathrm{a}}$ \\
\hline \multicolumn{4}{|l|}{ Macular status } \\
\hline Macula off, $n(\%)$ & $24(71)$ & $26(76)$ & \multirow[t]{2}{*}{$0.582^{\mathrm{a}}$} \\
\hline Macula on, $n(\%)$ & $10(29)$ & $8(24)$ & \\
\hline Vitreous haemorrhage, $n(\%)$ & $3(9)$ & $5(15)$ & $0.709^{a}$ \\
\hline \multicolumn{4}{|l|}{ Preoperative PVR } \\
\hline 0 or grade $\mathrm{A}, n(\%)$ & $13(38)$ & $11(32)$ & \multirow{3}{*}{$0.748^{\mathrm{a}}$} \\
\hline Grade $B, n(\%)$ & $10(29)$ & $9(27)$ & \\
\hline Grade C, $n(\%)$ & $11(33)$ & $14(41)$ & \\
\hline Combined cataract extraction, $n(\%)$ & $20(59)$ & $13(38)$ & $0.089^{a}$ \\
\hline During RRD surgery & $3(9)$ & $5(15)$ & $0.709^{c}$ \\
\hline During SOR & $17(50)$ & $8(24)$ & $0.024^{\mathrm{a}}$ \\
\hline ILM or ERM peeling, $n(\%)$ & $19(56)$ & $23(67)$ & $0.318^{\mathrm{a}}$ \\
\hline During RRD surgery & $16(47)$ & $18(53)$ & $0.628^{a}$ \\
\hline During SOR & $3(9)$ & $5(14)$ & $0.709^{c}$ \\
\hline Use of PFCL, $n(\%)$ & $16(42)$ & $21(62)$ & $0.223^{a}$ \\
\hline
\end{tabular}

SO, silicone oil; SOR, silicone oil removal; SD, standard deviation; IQR, interquartile range; RRD, rhegmatogenous retinal detachment; PVR, proliferative vitreoretinopathy; ILM, internal limiting membrane; $E R M$, epiretinal membrane; $P F C L$, perfluorocarbon liquid; $A L$, axial length. ${ }^{a} X^{2}$ test. ${ }^{b}$ Student's $t$ test. ${ }^{c}$ Fisher's exact test. ${ }^{d}$ Mann-Whitney $U$ test. ular status $(p=0.582)$, PVR grade $(p=0.748)$, and presence of vitreous haemorrhage $(p=0.709)$.

There were $23(68 \%)$ phakic eyes in group 1 and 13 (38\%) in group $2(p=0.015), 20$ and 13 of which underwent combined cataract extraction $(p=0.089)$. ERM removal and/or ILM peeling was performed during RRD surgery or SO extraction in $16(47 \%)$ and $3(9 \%)$ of eyes in group 1 and in $18(53 \%)$ and $5(14 \%)$ of eyes in group 2 , respectively $(p=0.628$ and $p=0.709)$.

\section{Effect of Tamponade Duration on Retinal Structure}

Table 2 indicates changes in retinal layer thickness and macular microstructure for both groups. The median tamponade duration was $4(3 ; 5)$ months in group 1 and $8(7 ; 9)$ months in group $2(p<0.001)$. After SOR, the median follow-up duration was $6(3 ; 14)$ and $7(4 ; 11)$ months in groups 1 and 2 , respectively $(p=0.602)$.
Both groups had comparable mean CMT, IRLT, and ORLT before SOR ( $p=0.732, p=0.286$ and $p=0.344$ ). The mean CMT significantly increased from $245.3 \pm 22.2$ $\mu \mathrm{m}$ and $238.8 \pm 41.6 \mu \mathrm{m}$ under $\mathrm{SO}$ to $281.3 \pm 60.2 \mu \mathrm{m}$ and $259.0 \pm 43.5 \mu \mathrm{m}$ after SOR in group $1(p=0.009)$ and in group $2(p=0.007)$, respectively. Interestingly, automated segmentation measurement revealed a significant increase in mean IRLT ( $p=0.014$ and $p=0.013$ ) but no significant change in mean ORLT $(p=0.080$ and $p=0.257)$ in both groups. After adjusting for differences in pre-SOR values, there was no difference between the 2 groups in terms of mean final CMT, IRLT, and ORLT ( $p=0.303$, $p=0.381$ and $p=0.500)$ and mean retinal thickness changes following SO extraction $(p=0.303, p=0.381$ and $p=0.500)$. Similarly, there was no correlation between tamponade duration and CMT, IRLT, and ORLT changes when considering the study population as a whole ( $p=$ $0.745, p=0.881$ and $p=0.731$ ). 
Table 2. Changes in retinal layer thickness and macular microstructure before and after SOR according to the tamponade duration

\begin{tabular}{|c|c|c|c|}
\hline & $\begin{array}{l}\text { Group } 1 \\
(n=34)\end{array}$ & $\begin{array}{l}\text { Group } 2 \\
(n=34)\end{array}$ & $p$ value \\
\hline Tamponade duration, months (median [IQR]) & $4[3 ; 5]$ & $8[7 ; 9]$ & $<0.001^{\mathrm{a}}$ \\
\hline Follow-up duration after SOR, months (median [IQR]) & $6[3 ; 14]$ & $7[4 ; 11]$ & $0.602^{a}$ \\
\hline \multicolumn{4}{|l|}{ Central retinal layer thickness before SOR, $\mu \mathrm{m}$ (mean $\pm \mathrm{SD})$} \\
\hline CMT & $245.3 \pm 22.2$ & $238.8 \pm 41.6$ & $0.732^{\mathrm{a}}$ \\
\hline IRLT & $163.4 \pm 19.4$ & $143.6 \pm 43.4$ & $0.286^{\mathrm{a}}$ \\
\hline ORLT & $80.0 \pm 11.1$ & $74.7 \pm 13.8$ & $0.344^{\mathrm{a}}$ \\
\hline \multicolumn{4}{|l|}{ Central retinal layer thickness after SOR, $\mu$ m (mean \pm SD) } \\
\hline CMT & $281.3 \pm 60.2$ & $259.0 \pm 43.5$ & $0.303^{b}$ \\
\hline IRLT & $198.9 \pm 66.3$ & $173.2 \pm 44.5$ & $0.381^{b}$ \\
\hline ORLT & $84.3 \pm 3.7$ & $78.1 \pm 20.5$ & $0.500^{\mathrm{b}}$ \\
\hline \multicolumn{4}{|c|}{ Central retinal layer thickness changes, $\mu \mathrm{m}$ (mean $\pm \mathrm{SD}$ ) } \\
\hline CMT & $36.0 \pm 54.8$ & $20.2 \pm 28.2$ & $0.303^{b}$ \\
\hline IRLT & $37.8 \pm 65.6$ & $26.2 \pm 38.2$ & $0.381^{b}$ \\
\hline ORLT & $4.6 \pm 10.3$ & $3.0 \pm 18.1$ & $0.500^{\mathrm{b}}$ \\
\hline \multicolumn{4}{|l|}{ Microstructural macular changes under SO, $n(\%)$} \\
\hline EZ disruption & $12(35)$ & $24(71)$ & $0.004^{c}$ \\
\hline ELM disruption & $8(24)$ & $16(47)$ & $0.042^{c}$ \\
\hline CME & $6(18)$ & $7(21)$ & $0.758^{c}$ \\
\hline ERM & $3(9)$ & $5(15)$ & $0.709^{d}$ \\
\hline PSF & $3(9)$ & $3(9)$ & $1^{d}$ \\
\hline \multicolumn{4}{|l|}{ Microstructural macular changes after SOR, $n(\%)$} \\
\hline EZ disruption & $9(27)$ & $13(38)$ & $0.300^{c}$ \\
\hline ELM disruption & $5(15)$ & $9(27)$ & $0.230^{c}$ \\
\hline CME & $5(15)$ & $2(6)$ & $0.427^{d}$ \\
\hline ERM & $8(24)$ & $6(18)$ & $0.549^{c}$ \\
\hline PSF & $1(3)$ & 0 & NC \\
\hline
\end{tabular}

IQR, interquartile range; SO, silicone oil; SOR, silicone oil removal; CMT, central macular thickness; IRLT, inner retinal layer thickness; ORLT, outer retinal layer thickness; SD, standard deviation; EZ, ellipsoid zone; ELM, external limiting membrane; ERM, epiretinal membrane; CME, central macular oedema; PSF, persistent subfoveolar fluid; NC, no computed. ${ }^{\text {a }}$ MannWhitney $U$ test. ${ }^{b}$ Covariance analysis with pre-SOR values used as the covariate. ${ }^{c} X^{2}$ test. ${ }^{d}$ Fisher's exact test.
Analysis of the macular microstructure showed that eyes with longer tamponade duration exhibited greater photoreceptor layer disruption before SOR. However, both groups had comparable EZ and ELM alterations at the end of the follow-up ( $p=0.300$ and $p=0.230$ ). CME, ERM, and PSF were detected during the tamponade period or after SOR in $11(32 \%), 11(32 \%)$ and $4(12 \%)$ of eyes in group 1 and in $9(27 \%), 11(32 \%)$, and $3(9 \%)$ of eyes in group 2; none of these differences were statistically significant $(p=0.595, p=1$ and $p=0.690)$.

Eighteen (53\%) eyes in group 1 and 24 (71\%) eyes in group 2 underwent SD-OCT within the first month after RRD surgery $(p=0.134)$. The mean CMT, IRLT, and ORLT were similar in both subgroups at baseline ( $p=$ $0.279, p=0.131$ and $p=0.343$ ) and at the last visit under SO after adjusting for baseline values and macular complications $(p=0.416, p=0.159$ and $p=0.357)$. Likewise, we did not observe any correlation between mean CMT, IRLT, and ORLT changes and tamponade duration in the 42 patients who had $2 \mathrm{SD}$-OCT examinations under SO ( $p=0.478, p=0.827$ and $p=0.209$ ). Interestingly, subgroup analysis of eyes without ERM, CME, or PSF demonstrated a thinning of all retinal layers, mainly affecting the IRL that resolved following SO extraction in both subgroups.

\section{Effect of Tamponade Duration on Visual Acuity}

Functional outcomes for both groups are summarized in Table 3. Twenty-two of 23 (96\%) eyes in group 1 and 13 of $13(100 \%)$ eyes in group 2 underwent subsequent cataract removal. At the end of the follow-up, 33 (97\%) and $34(100 \%)$ eyes were pseudophakic in groups 1 and 2 , respectively $(p=1)$. 
Table 3. Changes in visual acuity before and after SOR according to the tamponade duration

\begin{tabular}{lllr}
\hline & Group 1 $(n=34)$ & Group 2 $(n=34)$ & $p$ value \\
\hline Tamponade duration, months (median [IQR]) & $4[3 ; 5]$ & $8[7 ; 9]$ & $<0.001^{\mathrm{a}}$ \\
Pre-SOR BCVA, logMAR (mean \pm SD) & $0.7 \pm 0.4$ & $1.0 \pm 0.6$ & $0.143^{\mathrm{a}}$ \\
Final BCVA, logMAR (mean \pm SD) & $0.6 \pm 0.4$ & $0.9 \pm 0.7$ & $0.219^{\mathrm{b}}$ \\
Visual improvement, logMAR (mean \pm SD) & $0.2 \pm 0.3$ & $0.1 \pm 0.4$ & $0.219^{\mathrm{b}}$ \\
Pseudophakic eyes at the end of the follow-up, $n(\%)$ & $33(97)$ & $34(100)$ & $1^{\mathrm{c}}$ \\
\hline
\end{tabular}

IQR, interquartile range; SOR, silicone oil removal; BCVA, best-corrected visual acuity, logMAR, logarithm of the minimum angle of resolution; SD, standard deviation. ${ }^{a}$ Mann-Whitney $\mathrm{U}$ test. ${ }^{\mathrm{b}}$ Covariance analysis with pre-SOR

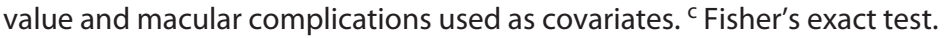

Both groups had comparable mean BCVA before SOR $(p=0.143)$. Group 1 exhibited a significant improvement in mean BCVA (from $0.7 \pm 0.4 \log \mathrm{MAR}$ under SOR to $0.6 \pm 0.4 \log \mathrm{MAR}, p=0.010$ ), while group $2 \mathrm{did}$ not (from $1.0 \pm 0.6 \log$ MAR to $0.9 \pm 0.7 \log \mathrm{MAR}, p=0.448)$. Nonetheless, after adjusting for pre-SOR values and macular complications, there was no difference between the 2 groups in mean final BCVA and mean visual changes $(p$ $=0.219$ and $p=0.219$ ). Similarly, covariance analysis did not reveal any significant correlation between BCVA changes and tamponade duration in the overall study population $(p=0.074)$.

\section{Complications}

One retinal redetachment occurred after SOR in group 2. We did not observe any cases of SO emulsification or elevated IOP exceeding $30 \mathrm{~mm} \mathrm{Hg}$. None of the patients experienced unexplained vision loss during SO tamponade or following SO extraction.

\section{Discussion}

In this study, we sought to design a retrospective comparative analysis to evaluate the impact of tamponade duration on SO-associated retinal changes following successful RRD surgery. We were able to define 2 balanced groups with significant difference in median tamponade duration $(4[3 ; 5]$ months vs. $8[7 ; 9]$ months). Both groups were comparable with respect to baseline characteristics (except for lens status), thereby reducing the effect of potential confounding variables.

Our analysis confirms that $\mathrm{SO}$ induces substantial structural changes in the retina, as evidenced by the significant increase in CMT after SOR in both groups. This is consistent with 2 recent studies which demonstrated that $\mathrm{SO}$ tam- ponade caused a transient thinning of central retinal layers that resolved following SO extraction $[18,26]$. By contrast, some authors reported that SO durably affected the retinal thickness, especially in eyes with macula-on RRD, suggesting a deleterious effect on retinal anatomy $[12,14,15,19$, $22]$. It is, however, very difficult to make comparisons across studies given the wide variations in inclusion criteria, surgical procedure, type of $\mathrm{SO}$, and time of SD-OCT assessment. Rabina et al. [18] using a design similar to ours, evaluated the change in CMT before and after SOR in eyes with successfully repaired RRD in comparison with the fellow healthy eyes. Consistent with our series, they found that SO tamponade induced a temporary decrease in CMT, mainly affecting the IRL [18].

There is a paucity of studies investigating the effect of tamponade duration on retinal thickness [15, 18, 22]. All failed to demonstrate any relationship and speculated that the tamponade period (3-6 months) was not long enough to uncover such an association. Our results show that the degree of retinal thinning under $\mathrm{SO}$ and thickness changes following SOR are not affected by longer tamponade (over 6 months). Interestingly, we noted that the retinal thickness values collected immediately after RRD surgery, when available, were comparable to those measured at the end of the follow-up, with no apparent difference between the 2 groups.

The reasons for retinal layer thinning with intraocular SO remain unclear. Possible causes include mechanical pressure on the retina induced by the SO bubble, retinal ionic environmental changes, and inflammatory reactions involving microglial cells and various cytokines [15, 17-19, 22]. Some authors also raised the possibility of retinal dehydration facilitated by the hydrophobic nature of SO [18]. Others suggested the subretinal migration of $\mathrm{SO}$ bubbles or diffusion of emulsified SO or low molecular weight components into the intraretinal space $[27,28]$. However, all pa- 
tients in the study received highly purified, solvent-free SO with a low concentration of potentially toxic elements. Besides, we did not observe any cases of prominent $\mathrm{SO}$ emulsification or migration of SO in the subretinal space on the SD-OCT images. Regardless of the underlying cause, our findings, taken together, suggest that the thinning effect of SO occurs at an early stage, probably within 4 months after $\mathrm{SO}$ injection and slowly progresses thereafter.

Similarly, the rate of ERM, CME, and PSF was not found to increase with a longer period of SO tamponade, at any time prior to or after SOR. Furthermore, we did not observe any correlation between the SO tamponade period and photoreceptor layer disruption postoperatively, suggesting that the duration of tamponade does not alter the photoreceptor recovery. These results stand in contrast with those of 2 previous studies which reported that eyes with prolonged tamponade were more prone to develop macular microstructural changes under SO and to exhibit photoreceptor damage following SO extraction $[20,21]$. However, contrary to our study, they did not consider baseline RRD characteristics and surgical manoeuvres in their analysis, which may have influenced their outcomes. Indeed, it has been demonstrated that the duration of macular detachment is a major factor affecting the ORL integrity after macula-off RRD repair [29]. Similarly, ILM peeling during RRD surgery has been shown to reduce the incidence of secondary ERM substantially [30]. It is therefore difficult to draw any definite conclusions regarding the microstructural changes without controlling for these 2 significant confounding variables. We believe that our series, with balanced and comparable groups, provides better insight into the influence of SO tamponade duration.

Finally and importantly, we assessed the correlation between the SO tamponade period and visual acuity. BCVA significantly improved following $\mathrm{SO}$ extraction in group 1 while it did not in group 2, which may be explained by the higher proportion of combined cataract extraction in group 1 . At the end of the follow-up, all eyes, except 1 in group 2, were pseudophakic and after adjusting for pre-SOR BCVA and postoperative complications, no difference was found between the 2 groups in terms of final BCVA or visual improvement. Previous studies reported discrepant results regarding the effect of tamponade duration on visual outcomes [2, 21-23]. However, again, it is difficult to make comparisons with the present series given that most of these studies did not consider potential confounders and notably, the macular status before surgery. In contrast, our findings are concordant with those of Lee et al. [22] who showed no correlation between the tamponade period and final BCVA after ad- justing for age, sex, and AL in patients undergoing PPV and SO tamponade for macula-on RRD.

We acknowledge several limitations to our study mainly related to its retrospective design and the relatively small sample size. Firstly, the indication for SO tamponade and the timing for SOR were left to the judgement of the surgeon, which may represent a selection bias. However, both groups had comparable baseline RRD characteristics and SD-OCT measurements, and we believe that this point is unlikely to have a major impact on our main findings. Secondly, preoperative SD-OCT images were not available in all patients and we were not able to evaluate whether final retinal thickness was a "restitutio ad integrum" of presurgical values, notably in maculaon RRD. Finally, the SD-OCT follow-up was not standardized, notably during the first months after RRD surgery, and we were not able to precisely examine the time course of SO-induced changes. In addition, the number of cases with early SOR (within 3 months after RRD surgery) was too low for valid statistical analysis, an analysis that would have been useful to consider examining the impact of SO tamponade duration clearly. Nonetheless, most surgeons recommend a minimum of 3-6 months of intraocular tamponade to reduce the risk of PVR redetachment, a process that has been shown to typically occur within 3 months after RRD repair [2, 5, 6, 31, 32].

In summary, our results confirm that $\mathrm{SO}$ tamponade causes a thinning of all retinal layers, mainly affecting the inner retinal layer. However, these changes resolved following SO extraction and were not affected by longer tamponade duration. The tamponade period also had no impact on the microstructural changes occurring before or after SOR and final visual acuity.

\section{Statement of Ethics}

All patients were given complete information on the risks and benefits of the surgical procedure and gave their written consent before surgery. The study adhered to the tenets of the Declaration of Helsinki, and the protocol was approved by the Ethics Committee of the French Society of Ophthalmology.

\section{Conflict of Interest Statement}

The authors have no conflicts of interest to declare.

\section{Funding Sources}

We did not receive any funding. 


\section{Author Contributions}

C.D. and J.-B.C. contributed to conception and design. C.D. and J.-B.C. contributed to data collection. J.S., C.D., and J.-B.C. contributed to acquisition, analysis, and interpretation of data. K.A.-D. and J.-P.B. contributed to review of the manuscript. J.-B.C. contributed to overall responsibility.

\section{Data Availability Statement}

The protocol and the datasets generated during and/or analysed during the current study are available from the corresponding author upon reasonable request.

\section{References}

1 Azen SP, Scott IU, Flynn HW, Lai MY, Topping TM, Benati L, et al. Silicone oil in the repair of complex retinal detachments. A prospective observational multicenter study. Ophthalmology. 1998 Sep;105(9):1587-97.

2 Rhatigan M, McElnea E, Murtagh P, Stephenson K, Harris E, Connell P, et al. Final anatomic and visual outcomes appear independent of duration of silicone oil intraocular tamponade in complex retinal detachment surgery. Int J Ophthalmol. 2018;11(1):83-8.

3 Vaziri K, Schwartz SG, Kishor KS, Flynn HW. Tamponade in the surgical management of retinal detachment. Clin Ophthalmol. 2016; 10:471-6.

4 Federman JL, Schubert HD. Complications associated with the use of silicone oil in 150 eyes after retina-vitreous surgery. Ophthalmology. 1988 Jul;95(7):870-6.

5 Jiang Y, Li X. The best timing of silicone oil removal. Zhonghua Yan Ke Za Zhi. 1997 Jan; 33(1):39-41.

6 Zilis JD, McCuen BW, de Juan E, Stefansson E, Machemer R. Results of silicone oil removal in advanced proliferative vitreoretinopathy. Am J Ophthalmol. 1989 Jul;108(1):1521.

7 Hutton WL, Azen SP, Blumenkranz MS, Lai MY, McCuen BW, Han DP, et al. The effects of silicone oil removal. Silicone study report 6. Arch Ophthalmol. 1994 Jun;112(6):77885.

8 Scheerlinck LM, Schellekens PA, Liem AT, Steijns D, van Leeuwen R. Incidence, risk factors, and clinical characteristics of unexplained visual loss after intraocular silicone oil for macula-on retinal detachment. Retina. 2016 Feb;36(2):342-50.

9 Newsom RS, Johnston R, Sullivan PM, Aylward GB, Holder GE, Gregor ZJ. Sudden visual loss after removal of silicone oil. Retina. 2004 Dec;24(6):871-7.

10 Williams PD, Fuller CG, Scott IU, Fuller DG, Flynn HW. Vision loss associated with the use and removal of intraocular silicone oil. Clin Ophthalmol. 2008 Dec;2(4):955-9.

11 Moya R, Chandra A, Banerjee PJ, Tsouris D, Ahmad N, Charteris DG. The incidence of unexplained visual loss following removal of silicone oil. Eye. 2015 Nov;29(11):1477-82.

12 Christensen UC, la Cour M. Visual loss after use of intraocular silicone oil associated with thinning of inner retinal layers. Acta Ophthalmol. 2012 Dec;90(8):733-7.
13 Avitabile T, Bonfiglio V, Sanfilippo M, Torrisi B, Reibaldi A. Correlation of optical coherence tomography pattern and visual recovery after vitrectomy with silicone oil for retinal detachment. Retina. 2006 Oct;26(8): 917-21.

14 Caramoy A, Droege KM, Kirchhof B, Fauser S. Retinal layers measurements in healthy eyes and in eyes receiving silicone oil-based endotamponade. Acta Ophthalmol. 2014 Jun; 92(4):e292-7.

15 Purtskhvanidze K, Hillenkamp J, Tode J, Junge O, Hedderich J, Roider J, et al. Thinning of inner retinal layers after vitrectomy with silicone oil versus gas endotamponade in eyes with macula-off retinal detachment. Ophthalmologica. 2017;238(3):124-32.

16 Saber EE, Bayoumy ASM, Elmohamady MN, Faramawi HM. Macular microstructure and visual acuity after macula-off retinal detachment repair by 23 -gauge vitrectomy plus silicone endotamponade. Clin Ophthalmol. 2018;12:2005-13.

17 Jurišić D, Geber MZ, Ćavar I, Utrobičić DK. Retinal layers measurements following silicone oil tamponade for retinal detachment surgery. Semin Ophthalmol. 2018;33(5):7118.

18 Rabina G, Azem N, Barequet D, Barak A, Loewenstein A, Schwartz S. Silicone oil tamponade effect on macular layer thickness and visual acuity. Retina. 2020 May;40(5):9981004.

19 Inan S, Polat O, Ozcan S, Inan UU. Comparison of long-term automated retinal layer segmentation analysis of macula between silicone and gas tamponade after vitrectomy for rhegmatogenous retinal detachment. Ophthalmic Res. 2020;63(6):524-32.

20 Bae SH, Hwang JS, Yu HG. Comparative analysis of macular microstructure by spectraldomain optical coherence tomography before and after silicone oil removal. Retina. 2012 Oct;32(9):1874-83.

21 Durrani AK, Rahimy E, Hsu J. Outer retinal changes on spectral-domain optical coherence tomography pre- and post-silicone oil removal. Ophthalmic Surg Lasers Imaging Retina. 2017;48(12):978-82.

22 Lee SH, Han JW, Byeon SH, Kim SS, Koh HJ, Lee SC, et al. Retinal layer segmentation after silicone oil or gas tamponade for macula-on retinal detachment using optical coherence tomography. Retina. 2018 Feb;38(2):310-9.
23 Eibenberger K, Sacu S, Rezar-Dreindl S, Schmidt-Erfurth U, Georgopoulos M. Silicone oil tamponade in rhegmatogenous retinal detachment: functional and morphological results. Curr Eye Res. 2020;45(1):38-45.

24 Machemer R, Aaberg TM, Freeman HM, Irvine AR, Lean JS, Michels RM. An updated classification of retinal detachment with proliferative vitreoretinopathy. Am J Ophthalmol. 1991 Aug;112(2):159-65.

25 Staurenghi G, Sadda S, Chakravarthy U, Spaide RF, International nomenclature for optical coherence tomography (IN OCT) panel. Proposed lexicon for anatomic landmarks in normal posterior segment spectraldomain optical coherence tomography: the IN OCT consensus. Ophthalmology. 2014 Aug;121(8):1572-8.

26 Lo DM, Flaxel CJ, Fawzi AA. Macular effects of silicone oil tamponade: optical coherence tomography findings during and after silicone oil removal. Curr Eye Res. 2017;42(1): 98-103.

27 Miller JB, Papakostas TD, Vavvas DG. Complications of emulsified silicone oil after retinal detachment repair. Semin Ophthalmol. 2014 Nov;29(5-6):312-8.

28 Gabel VP, Kampik A, Burkhardt J. Analysis of intraocularly applied silicone oils of various origins. Graefes Arch Clin Exp Ophthalmol. 1987;225(3):160-2.

29 Malosse L, Rousseau H, Baumann C, Angioi K, Leroy B, Sennlaub F, et al. Prevalence and risk factors for outer retinal layer damage after macula-off retinal detachment. Br J Ophthalmol. 2020 May;104(5):660-5.

30 Fallico M, Russo A, Longo A, Pulvirenti A, Avitabile T, Bonfiglio V, et al. Internal limiting membrane peeling versus no peeling during primary vitrectomy for rhegmatogenous retinal detachment: a systematic review and meta-analysis. PLoS One. 2018;13(7): e0201010.

31 Casswell AG, Gregor ZJ. Silicone oil removal. II. Operative and postoperative complications. Br J Ophthalmol. 1987 Dec;71(12):898902.

32 Gonvers M. Temporary silicone oil tamponade in the management of retinal detachment with proliferative vitreoretinopathy. Am J Ophthalmol. 1985 Aug;100(2):239-45.
Effect of Silicone Oil Tamponade on Retina after RRD Surgery
Ophthalmologica 2022;245:144-151 DOI: $10.1159 / 000519520$ 